\title{
Narrow Precursor Mass Range for DIA-MS Enhances Protein Identification and Quantification in Arabidopsis
}

\author{
Huoming Zhang (D) and Dalila Bensaddek * \\ Core Labs, King Abdullah University of Science and Technology, Thuwal 23500-6900, Saudi Arabia; \\ huoming.zhang@kaust.edu.sa \\ * Correspondence: dalila.bensaddek@kaust.edu.sa
}

Citation: Zhang, H.; Bensaddek, D Narrow Precursor Mass Range for DIA-MS Enhances Protein Identification and Quantification in Arabidopsis. Life 2021, 11, 982. https://doi.org/10.3390/life11090982

Academic Editors: Marco Chiapello, Nino Nikolovski and Claudius Marondedze

Received: 30 June 2021

Accepted: 15 September 2021

Published: 18 September 2021

Publisher's Note: MDPI stays neutral with regard to jurisdictional claims in published maps and institutional affiliations.

Copyright: (c) 2021 by the authors. Licensee MDPI, Basel, Switzerland. This article is an open access article distributed under the terms and conditions of the Creative Commons Attribution (CC BY) license (https:/ / creativecommons.org/licenses/by/ $4.0 /)$.

\begin{abstract}
Data independent acquisition-mass spectrometry (DIA-MS) is becoming widely utilised for robust and accurate quantification of samples in quantitative proteomics. Here, we describe the systematic evaluation of the effects of DIA precursor mass range on total protein identification and quantification. We show that a narrow mass range of precursors $(\sim 250 \mathrm{~m} / \mathrm{z})$ for DIA-MS enables a higher number of protein identifications. Subsequent application of DIA with narrow precursor range (from 400 to $650 \mathrm{~m} / \mathrm{z}$ ) on an Arabidopsis sample with spike-in known proteins identified $34.7 \%$ more proteins than in conventional DIA (cDIA) with a wide precursor range of $400-1200 \mathrm{~m} / \mathrm{z}$. When combining several DIA-MS analyses with narrow precursor ranges (i.e., 400-650, 650-900 and $900-1200 \mathrm{~m} / \mathrm{z}$ ), we were able to quantify 10,099 protein groups with a median coefficient of variation of $<6 \%$. These findings represent a $54.7 \%$ increase in the number of proteins quantified than with cDIA analysis. This is particularly important for low abundance proteins, as exemplified by the six-protein mix spike-in. In cDIA only five out of the six-protein mix were quantified while our approach allowed accurate quantitation of all six proteins.
\end{abstract}

Keywords: data-independent acquisition (DIA); mass spectrometry; precursor mass range selection; Arabidopsis; quantitative proteomics

\section{Introduction}

Mass spectrometry (MS)-based proteomics has become a method of choice to study complex biological systems in the last decade [1]. Quantitative proteomics strategies that can simultaneously identify and quantify a large number of proteins not only contribute to our understanding of biological systems at the molecular level but also can be used in biomarker discovery [2,3] and different stages in the drug discovery process [4-6]. Common quantitative proteomics strategies include isotopic labeling [7-9] and label-free approaches $[1,10]$. In these approaches, data dependent acquisition (DDA) is often used to isolate and fragment peptides of top-ranked intensity (e.g., top 20) as they elute from the liquid chromatography (LC) separation column directly into the MS sampling region. The peptide ions are selected for fragmentation using isolation windows of less than two Thomson depending on the instrument capabilities. Such methods are generally high-throughput but suffer from limited reproducibility of measurement, especially for peptides of relatively low abundance. This is due to the nature of DDA experiments, where peptide selection is largely stochastic. In addition, these experiments often require off-line fractionation prior to online LC-MS analysis in order to achieve an in-depth proteomics coverage, thus increasing the instrument time requirement. This, coupled with the expensive isotopic reagents for multiplexing in the case of TMT/iTRAQ, makes DDA cost ineffective, not to mention the issue of ratio compression in which the experimentally measured fold change is smaller than the anticipated or actual fold change between samples, especially for protein expression with a relatively large fold change [11,12]. In the case of label-free DDA analyses, it is very important to control the LC reproducibility especially for large numbers of samples. Targeted quantitative proteomics $[12,13]$, on the other hand, measures 
a small number of known proteins/peptides with higher sensitivity and reproducibility but it has limited use for proteome-wide survey.

Data-independent acquisition (DIA/SWATH) MS-based proteomics was developed more recently with the advantages of high reproducibility and accurate quantitation in a high-throughput fashion [14]. In contrast to DDA-MS, DIA-MS cyclically fragments all peptides across the entire precursor mass range (typically $400-1200 \mathrm{~m} / \mathrm{z}$ ). In each cycle, a large isolation window (e.g., $25 \mathrm{~m} / \mathrm{z}$ units) is used to sequentially isolate peptides from the defined precursor mass range to be fragmented. Typically, this is achieved using 32 windows and $2-3 \mathrm{~s}$ cycling time in most current mass spectrometers. Since a peak normally elutes from the LC within 15-30 s, a total of 5-10 MS data points can be collected and a peak profile can be well constructed for each peptide, thus enabling accurate peptide quantitation. It should be noted that DIA isolates and fragments a group of precursors using a wide window, thereby generating highly complex fragment ion spectra from a complex mixture (e.g., tissue or cell lysate). Subsequent targeted extraction of individual peptide spectra can be performed by matching with a preconstructed ion library $[15,16]$, an in silico library [17] or using a library-free approach [18].

The sensitivity of DIA-MS relies heavily on the fragment ion spectral quality, with higher resolution and less complexity of spectra significantly enhancing identifications. Significant improvements were reported by Bruderer et al. [19]; the authors combined high resolution acquisition in both MS1 and MS2, high sample loading with indexed retention time (iRT) peptides spike-in for library generation along with improved algorithm for data analysis to quantify $\sim 7100$ proteins from HEK-293 cells and $>8000$ proteins from a mouse brain tissue using single shot DIA-MS. Similarly, reducing the complexity of DIA spectra through a variable-window isolation strategy to ensure a similar number of precursors are isolated for each DIA fragmentation window across the defined precursor mass range was also shown to enhance protein identification by about 10\% [20]. Amodei et al. used overlapping DIA windows, which significantly improved the specificity of fragment ions and thus the sensitivity in detection [21]. Borras et al. developed a DIA+, which combined signals from identical peptides with different charge states through multiplexing scans. This resulted in an improved signal-to-noise, an additional number of fragments and a reduction in the number of analytes per composite window, thereby increasing the identification and quantification of peptides in complex samples [22]. More recently, Cai et al. reported a PulseDIA method in which they combined several DIA-MS analyses for the same sample with complementary narrow DIA isolation windows. As such, they were able to identify $29 \%$ more proteins [23]. Furthermore, using a small sample specific library was shown to reduce the false discovery rate (FDR) in the spectral match and improve in protein identification and quantification [24,25]. Certainly, with advances in MS technology, either with faster scan rates and incorporation of additional separation dimensions such as ion mobility, we would expect to be able to quantify high numbers of proteins in a single shot. For example, using a timsTOF pro in diaPASEF mode enabled the quantitation of more than 7000 proteins in 120 min gradient [26], while FAIMS Orbitrap was used to quantify over 8000 proteins with 6 h gradient [27].

Arabidopsis is one of the most important model organisms. It is the first plant to have its genome fully sequenced. It is a widely used a model organism for plant research. Earlier, we created a comprehensive, high quality Arabidopsis mass spectral library that is useful for DIA-MS proteomics quantitation [16]. Since the majority of peptides in the library fall within a narrow mass range (e.g., $\sim 60 \%$ in $400-700 \mathrm{~m} / \mathrm{z}$ ), we hypothesized that using a small precursor range for DIA-MS would result in an increase in resolution, ion specificity and match against small spectral library which in turn would increase overall identification without compromising protein quantitation accuracy. To this end, we systematically evaluated the effect of precursor mass range on protein identification and quantitation accuracy using Arabidopsis samples. We show here that it is possible to obtain a $34.7 \%$ increase in the number of protein groups quantified from a single injection with narrow precursor mass range (with size of $250 \mathrm{~m} / \mathrm{z}$ units) and a $54.7 \%$ increase 
when combing three DIA-MS analyses with narrow precursor mass ranges obtained using gas-phase fractionation.

\section{Materials and Methods}

\subsection{Arabidopsis Root Cell Suspension Culture}

Cells isolated from roots of Arabidopsis were grown in Gamborg's B5 basal salt mixture (Sigma-Aldrich, St. Louis, MO, USA) with 2,4-dichlorophenoxyacetic acid (2,4-D; $\left.1 \mathrm{mg} \mathrm{mL}^{-1}\right)$ and kinetin $\left(0.05 \mu \mathrm{g} \mathrm{mL}^{-1}\right)$ in sterile flask as described [28]. The cells were harvested by draining off the media using a Stericup ${ }^{\circledR}$ filter unit (Millipore, Billerica, MA, USA) and immediately flash frozen in liquid nitrogen and stored at $-80^{\circ} \mathrm{C}$ until use.

\subsection{Protein Extraction and Digestion}

The plant cells were ground in liquid nitrogen with a prechilled mortar and a pestle. The fine powder was resuspended in the extraction buffer $(50 \mathrm{~mm}$ Tris, $\mathrm{pH}$ 8, $8 \mathrm{M}$ urea and $0.5 \%$ SDS) supplemented with protease inhibitor (Roche Diagnostics GmbH, Mannheim, Germany) and homogenized with a Dounce homogenizer. Subsequently, the crude homogenate was subjected to 30 cyclic high/low pressurization (50 sec of 35,000 PSI and $10 \mathrm{sec}$ of ambient pressure) using a pressure cycling technology (Barocycle, PressureBioSciences, MA, USA). The extracts were then centrifuged at $10,000 \mathrm{~g}$ for $5 \mathrm{~min}$ at $4{ }^{\circ} \mathrm{C}$. The proteins in the supernatant were purified using methanol/chloroform precipitation and dried under vacuum. The dried pellets were dissolved into the extraction buffer with the aid of sonication. The protein content was determined using a microBCA kit (Thermo Scientific, Waltham, MA, USA). Approximately $100 \mu \mathrm{g}$ of proteins were reduced, alkylated and digested with trypsin using a FASP method [29]. The digests were desalted prior to LC-MS analysis with microcolumns packed with C18 material. To prepare protein digests from 6 proteins (Glycoprotein standards set, Thermo Scientific) for spike-in experiment, $10 \mu \mathrm{g}$ of each protein was dissolved into $50 \mathrm{mM}$ ammonium bicarbonate, reduced and alkylated prior to trypsin digestion as described [30].

\subsection{Mass Spectrometric Analysis Using Data Independent Acquisition (DIA) Mode}

The DIA-MS analysis was conducted using an Orbitrap Fusion Lumos mass spectrometer (Thermo Scientific) coupled with an UltiMate ${ }^{\mathrm{TM}} 3000$ UHPLC (Thermo Scientific). Approximately $0.8 \mu \mathrm{g}$ of peptide mixture was injected into a precolumn (Acclaim PepMap, $300 \mu \mathrm{m} \times 5 \mathrm{~mm}, 5 \mu \mathrm{m}$ particle size) and desalted for $15 \mathrm{~min}$ with $0.1 \%$ FA in water at a flow rate of $5 \mu \mathrm{L} / \mathrm{min}$. The peptides were eluted into an EasySpray C18 column $(50 \mathrm{~cm} \times 75 \mu \mathrm{m}$ ID, PepMap C18, $2 \mu \mathrm{m}$ particles, $100 \AA$ pore size, Thermo Scientific) and separated with a 130-min gradient at constant $300 \mathrm{~nL} / \mathrm{min}$, at $40{ }^{\circ} \mathrm{C}$. The gradient was established using mobile phase $\mathrm{A}\left(0.1 \% \mathrm{FA}\right.$ in $\left.\mathrm{H}_{2} \mathrm{O}\right)$ and mobile phase $\mathrm{B}\left(0.1 \% \mathrm{FA}, 95 \% \mathrm{ACN}\right.$ in $\left.\mathrm{H}_{2} \mathrm{O}\right)$ : 2.1-5.3\% B for $5 \mathrm{~min}, 5.3-10.5 \%$ for $15 \mathrm{~min}, 10.5-21.1 \%$ for $70 \mathrm{~min}, 21.1-31.6 \% \mathrm{~B}$ for $18 \mathrm{~min}$, ramping from 31.6 to $94.7 \% \mathrm{~B}$ in $2 \mathrm{~min}$, maintaining at $94.7 \%$ for $5 \mathrm{~min}$, and $4.7 \%$ B for 15-min column conditioning. The sample was introduced into the Fusion Lumos through an EasySpray (Thermo Scientific) with an electrospray potential of $1.9 \mathrm{kV}$. The ion transfer tube temperature was set at $270{ }^{\circ} \mathrm{C}$. The MS parameters included application mode as standard for peptide, RF lens as 30\%, default charge state of 3 and the use of EASY-IC as internal mass calibration in both precursor ions (MS1) and fragment ions (MS2). Other parameters for MS1 include a resolution of 60,000 (at $200 \mathrm{~m} / \mathrm{z}$ ), a maximum ion accumulation time of 50 milliseconds, a target value of 2e5, and data type of profile. For the MS2 by DIA, the mass defect was 0.9995 . The normalized HCD collision energy was set to $30 \%$ for peptide fragmentation. MS2 spectra were recorded in centroid mode at 30,000 resolution from 350-1500 m/z, with an AGC target of $1 \mathrm{e} 6$ and a maximum ion accumulation time of $100 \mathrm{~ms}$. To determine an optimal precursor window size for quantifying a higher number of protein groups, the sizes of precursor mass range from 150 to 800 (m/z unit) (Figure 1a) were evaluated for DIA-MS analysis. Accordingly, a quadrupole isolation window size 
was set from 4-20 (m/z unit) for each defined precursor mass range in order to achieve a similar number of total scan events $(n=39-41)$ per cycle.

In order to compare the identification and quantification between a conventional DIA (cDIA) and the DIA with optimal precursor scanning range, the 6 standard protein digests were spiked into the $1 \mu \mathrm{g}$ Arabidopsis peptides as detailed in Table 1 . In cDIA, a precursor mass ranges from 400 to $1200(\mathrm{~m} / \mathrm{z})$, with an isolation window of $25 \mathrm{~m} / \mathrm{z}$ and a total of 32 scan events. For the DIA with the optimal precursor scanning range as determined as above, we combined DIA with a gas-phased fractionation (GPF) strategy which divides precursor mass-to-charge ranges into several smaller precursor mass ranges for DIA-MS analysis. As such three independent DIA-MS experiments were performed for the same sample with precursor mass ranges 400-650; 650-900 and 900-1200 m/z, respectively. The isolation windows were 8 (for precursors 400-650 and 650-900 m/z) and $9 \mathrm{~m} / \mathrm{z}$ (for 900-1200 m/z) which resulted in a total of 32 and 31 scan events per cycle accordingly. All other parameters were the same as aforementioned. Each experiment was conducted in triplicate.

Table 1. Six standard protein digests were spiked into an equal amount of Arabidopsis peptide mixture with two different ratios to get sample 1 (S1) and sample 2 (S2).

\begin{tabular}{cccccccccc}
\hline \multicolumn{3}{c}{ Protein Information } & & $\begin{array}{c}\text { Quantity (fmol) and Ratio } \\
\text { of Spike-In }\end{array}$ & \multicolumn{2}{c}{$\begin{array}{c}\text { Quantitation } \\
\text { by cDIA* }\end{array}$} & $\begin{array}{c}\text { Quantitation } \\
\text { by cGPF-DIA** }\end{array}$ \\
\hline & $\begin{array}{c}\text { SwissProt } \\
\text { Accession }\end{array}$ & $\begin{array}{c}\text { Molecular } \\
\text { Weight (Da) }\end{array}$ & S1 & S2 & S1:S2 & S1:S2 & Q-value & S1:S2 & Q-value \\
\hline BSA & P02769 & 69300 & 64.1 & 8.0 & $8: 1$ & $6.6: 1$ & $2.5 \times 10^{-18}$ & $6.5: 1$ & $2.4 \times 10^{-12}$ \\
\hline Transferrin & P02787 & 77070 & 57.7 & 7.2 & $8: 1$ & $6.8: 1$ & $3.3 \times 10^{-112}$ & $7.2: 1$ & $7.0 \times 10^{-13}$ \\
\hline $\begin{array}{c}\text { alpha-acid } \\
\text { glycoprotein }\end{array}$ & Q3SZR3 & 23190 & 191.7 & 24.0 & $8: 1$ & $8.8: 1$ & $2.4 \times 10^{-26}$ & $7.5: 1$ & $7.7 \times 10^{-27}$ \\
\hline Lysozyme & P00689 & 16240 & 34.2 & 171.0 & $1: 5$ & $1: 5.8$ & $4.7 \times 10^{-33}$ & $1: 5.4$ & $1.7 \times 10^{-55}$ \\
\hline Fetuin & Q58D62 & 42670 & 13.0 & 65.1 & $1: 5$ & $1: 19.7$ & $1.2 \times 10^{-1}$ & $1: 6.1$ & $4.9 \times 10^{-7}$ \\
\hline Ovalbumin & P01012 & 42890 & 13.0 & 64.8 & $1: 5$ & $1: 5.5$ & $9.7 \times 10^{-78}$ & $1: 5.5$ & $6.6 \times 10^{-37}$ \\
\hline
\end{tabular}

${ }^{*}$ cDIA: conventional DIA; ** cGPF-DIA: combined gas-phase fractionation DIA. $Q$-value is a $p$-value that has been adjusted for the False

Discovery Rate (FDR) and a $Q$-value of 0.001 is the equivalent to an FDR cutoff of $0.1 \%$ for measuring significant quantitative change.

\subsection{DIA-MS Data Analysis Using Spectronaut}

\subsubsection{Library Generation}

All DIA-MS data files and the search archive of our published Arabidopsis library [16] were combined for library generation using Spectronaut Pulsar (version 14.10.201222.47784, Biognosys, Zurich, Switzerland). The protein database was the combination of a TAIR11 proteome sequence and the six spike-in sequences. The default settings for database match include: full specificity trypsin digestion, peptide length of between 7 and 52 amino acids and maximum missed cleavage of 2. Besides, lysine and arginine (KR) were used as special amino acids for decoy generation, and N-terminal methionine was removed during preprocessing of the protein database. Carbamidomethylation at cysteine was used as a fixed modification, protein $\mathrm{N}$-terminal acetylation and methionine oxidation were set as variable modifications. The false discovery rates (FDRs) were set as 0.01 for the peptidespectrum match (PSM), peptide and protein identification. The other Biognosys default spectral library filters include amino acid length of ion more than 2, ion mass-to-charge between 300 and $1800 \mathrm{Da}$ and minimum relative intensity of 5\%. The best 3-6 fragments per peptide were included in the library. The iRT calibration was required with minimum R-Square of 0.8 . 


\subsubsection{Quantitation Analysis}

The DIA data were analyzed by Spectronaut using both a library-based matching and directDIA approach. In the directDIA, a collection of pseudo-MS2 spectra was generated from the DIA data and directly used for database search and library construction. The resulting DIA data specific library was then used for targeted analyses of the same DIA data. In the library approach, the newly generated spectral library from Section 2.4.1 was used for spectral matching. In both approaches, data extraction was based on maximum intensity in both MS1 and MS2 spectra with relative mass tolerances of 10 and 20 ppm, respectively. Retention time window of Extracted ion Chromatogram (XIC) was set dynamically but with a correction factor of 0.5 to enhance specificity. The parameters in the calibration category include allowing source specific iRT calibration at automatic mode, precision iRT with local nonlinear regression and a mass tolerance of $40 \mathrm{ppm}$ for both MS1 and MS2 spectra. The Biognosys default settings were applied for both identification and quantification. These parameters were excluding duplicate assay; generation decoy based on mutated method at $10 \%$ of library size, and estimation of FDRs using q-value as 0.01 for both precursors and proteins. The $p$-value was calculated by the kernel-density estimator. Interference correction was activated and a minimum of 3 fragment ions and 2 precursor ions were kept for the quantitation. The area of extracted ion chromatogram (XIC) at MS2 level was used for quantitation. Peptide (stripped sequence) quantity was measured by the mean of 1-3 best precursors, and protein quantity was calculated accordingly by the mean of 1-3 best peptides. Local normalization strategy and q-value sparse selection were used for cross run normalization. To determine the differential abundance between samples, the major group (quantification settings) was used for the differential abundance grouping, and the precursor ion (quantification settings) was chosen in the smallest quantitative unit. In addition, an unpaired $t$-test with assuming equal variance, group-wise testing correction and clustering was carried out. Proteins with a fold-change of higher than 1.5 and a q-value of less than 0.001 were considered as differentially expressed proteins.

\section{Results}

\subsection{Effects of DIA Precursor Mass Range Selection on the Protein Identification}

In the Arabidopsis library, the precursors are distributed along the mass-to-charge range $(\mathrm{m} / \mathrm{z})$ of $375-1400$ with approximately $60 \%$ of precursors concentrated within the narrow region of $400-700 \mathrm{~m} / \mathrm{z}$. To systematically evaluate the effects of the size of precursor mass range on the number of protein identifications, we performed DIA analyses with various sizes of precursor mass range from 150 to 800 with $50 \mathrm{~m} / \mathrm{z}$ increments. The precursor scan range started from $550-700 \mathrm{~m} / \mathrm{z}(150 \mathrm{~m} / \mathrm{z}$ units) to $400-1200 \mathrm{~m} / \mathrm{z}(800 \mathrm{~m} / \mathrm{z}$ units) as shown in Figure 1a.

Each DIA-MS file was analyzed using Spectronaut software. Precursors, peptides and proteins with a $Q$-value $<0.01$ were considered as positive identification and used for statistical analyses. As can be seen in Figure 1, the total number of identified precursors from each DIA-MS analysis, initially, increased with the size of precursor scan range then plateaued at the size of 400 (Figure 1b). A similar trend was observed with the identified peptides but it reached a steady level at a slightly smaller precursor scanning size (300-350) (Figure 1c). In contrast, the highest number of protein groups $(n>8,250)$ was identified from $150-300$ precursor scanning $\mathrm{m} / \mathrm{z}$. The number dropped by $\sim 30 \%$ when wider precursor scanning ranges (700-800 m/z units) were included in DIA-MS (Figure 1d). 


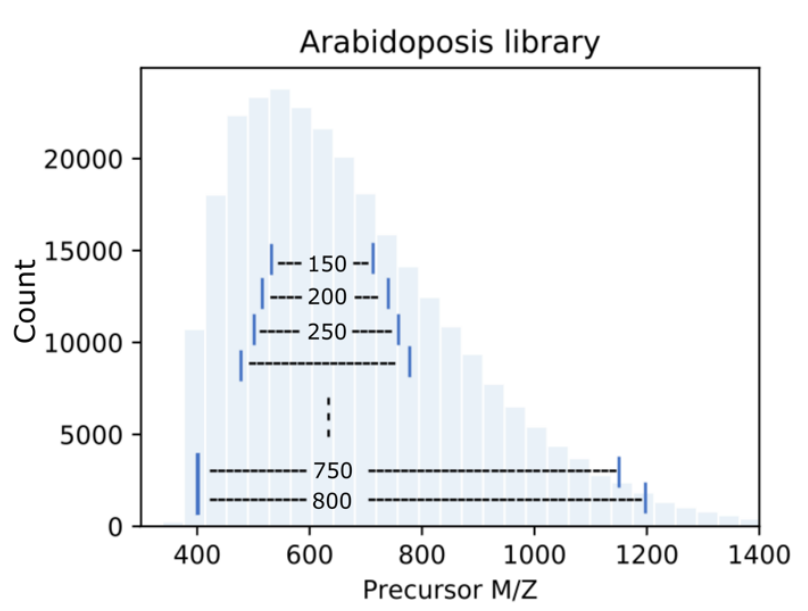

(a)

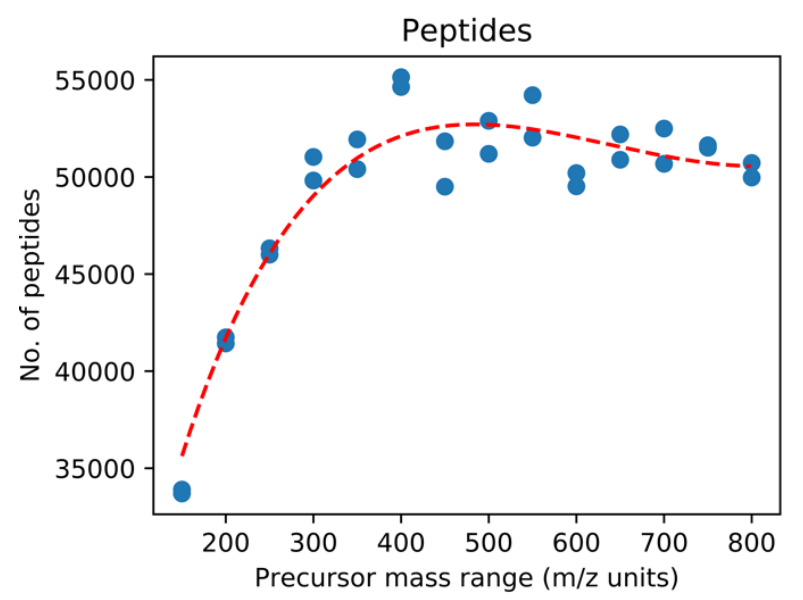

(c)

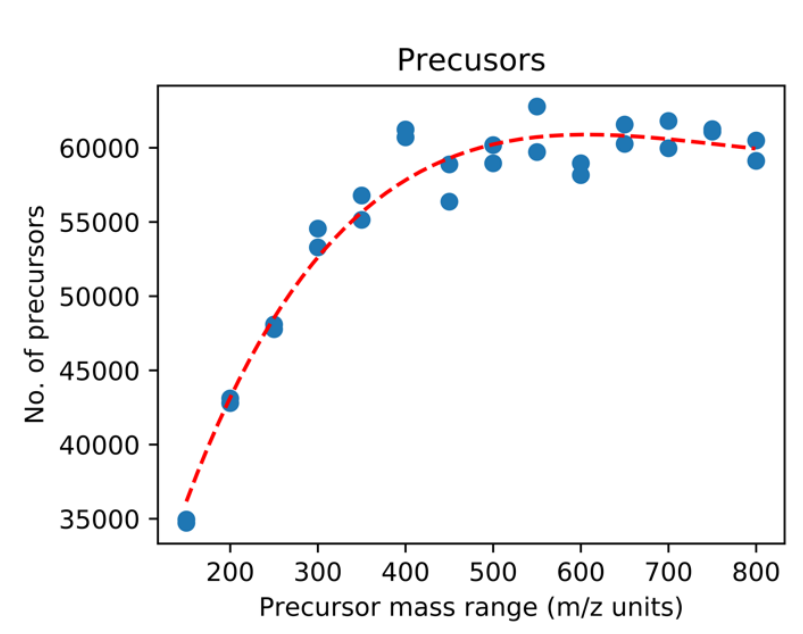

(b)

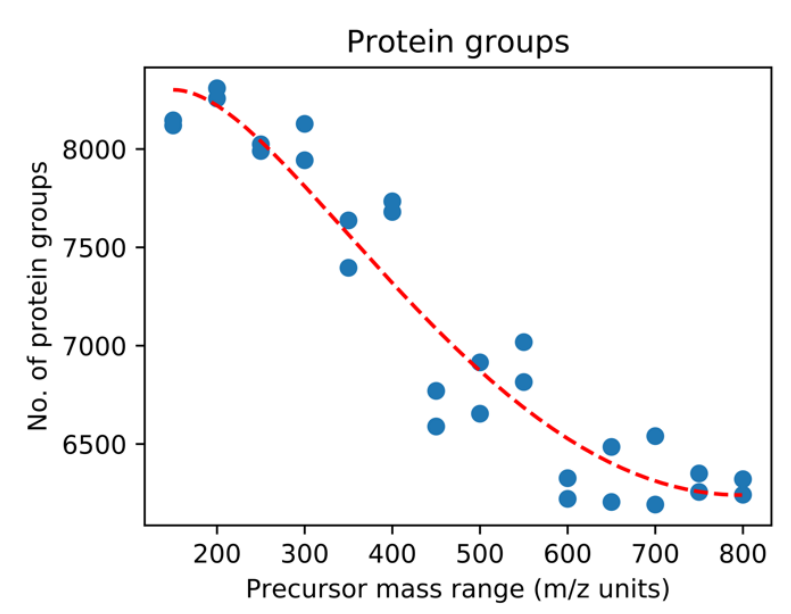

(d)

Figure 1. Size of precursor mass range affects DIA identification. (a) In the Arabidopsis precursor library, most of the precursors are distributed across the mass-to-charge range of 400-700 ( 62\%). The size of precursor $\mathrm{m} / \mathrm{z}$ ranges from 150 $(\mathrm{m} / \mathrm{z})$ to $800(\mathrm{~m} / \mathrm{z})$ with a step-up of $50(\mathrm{~m} / \mathrm{z})$ was systematically assessed for DIA-MS analysis. The experiment was conducted in duplicate. (b) The scatter plot shows that the number of precursors identified increases with the size of the precursor mass range selection. (c) The scatter plot shows that the number of identified peptides increases with the size of the precursor mass range selection. (d) The scatter plot shows that the number of protein groups identified decreases with size of the precursor mass range selection.

\subsection{Narrow Precursor Scan Range Quantified Higher Number of Proteins}

Having established an optimal size of precursor mass range of 200-300 (m/z), we combined DIA experiments with gas phase fractionation (GPF), where a wide precursor range used in conventional DIA (cDIA: 400-1200 m/z) was divided into precursor scan ranges: 400-650, 650-900 and 900-1200 m/z for three independent DIA-MS experiments respectively. Consistent with the fact that although narrow precursor scan range resulted in relatively lower peptide identifications (Figures $1 \mathrm{c}$ and $2 \mathrm{c}$ ), both narrow precursor range GPF-DIA analyses (with 400-650 and 650-900 m/z, respectively) resulted in the identification of higher numbers of protein groups $(n=8798$ and 8581 , respectively) compared to the cDIA $(n=6530)$ with 34.7 and $31.4 \%$ increase, respectively, whereas the GPF 900-1200 $(n=6344)$ identified similar number of protein groups (Figure 2a, Tables S1-S4) despite a much lower number of precursors in this mass range. The ratios of common identifications over total proteins from the triplicate analysis were all above $88 \%$, with highest of $97 \%$ in 
cDIA method. The missing values of protein groups (defined as not consistently identified in all replicate analyses) slightly increased in GPF-DIA likely due to the contribution from much lower abundance proteins. Overall, our data showed high reproducibility in identifying proteins across the full dynamic range for 8798 proteins from our GPF-DIA.

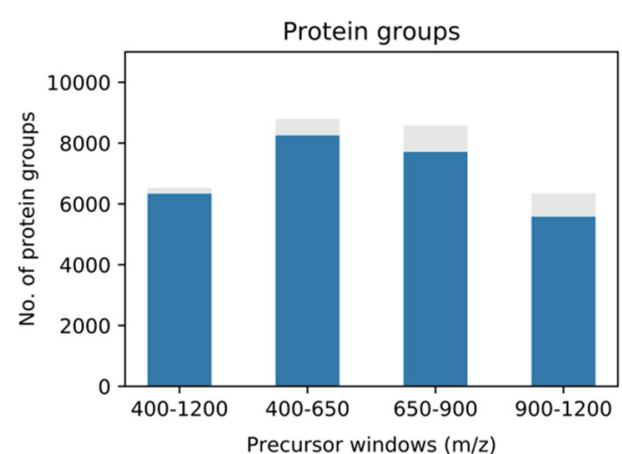

(a)

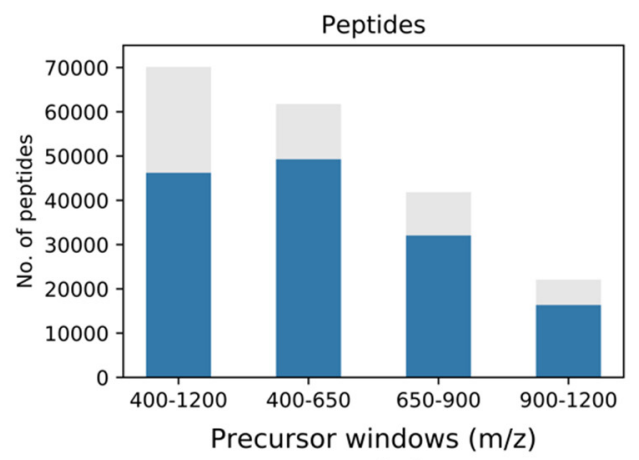

(c)

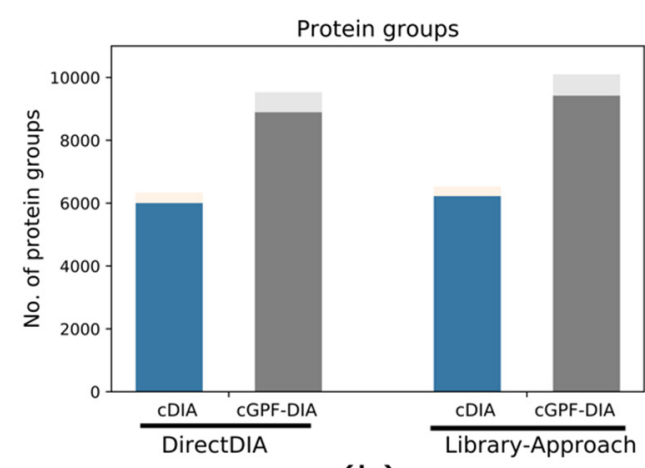

(b)

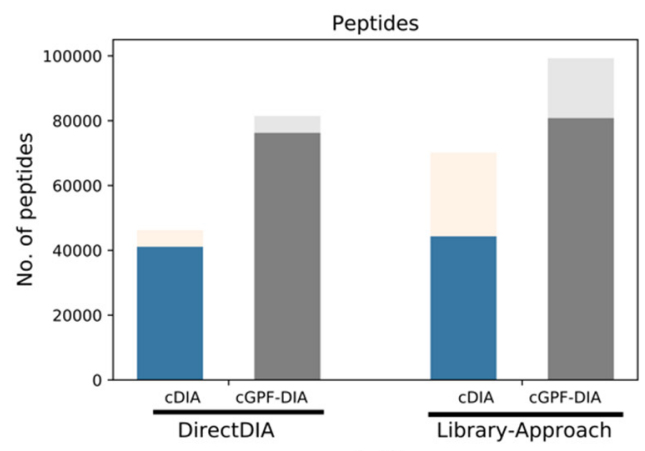

(d)

Figure 2. Protein groups and peptides identified from different approaches with each condition run in triplicate. The total number of protein groups (a) and peptides (c) identified from DIA-MS analyses of four different precursor mass ranges, respectively (cDIA: 400-1200 m/z, and three individual gas-phase fractionation (GPF)) by matching spectral library. Lower blue bar indicates common identification from the triplicate experiment, and upper gray bar shows the identification from 1 or 2 replicates only; whereas the total number of protein groups $(\mathbf{b})$ and peptides (d) identified from cDIA and the combination of three GPF-DIA (cGPF-DIA) via directDIA (left panel) and library-based approaches (right panel), respectively. CGPF-DIA identified $>45 \%$ more proteins and $>40 \%$ more peptides than cDIA in both directDIA and library-based data analysis approaches. Library-based approach obtained a modest increase in protein identification compared to directDIA. Lower dark color segment indicates common identification from the triplicate analysis whereas the upper light color bar shows the identification from 1 or 2 replicates only.

The benefit of using narrow precursor windows becomes more obvious when combining the three GPF-DIA runs for analysis (Figure 2b). A total of 10,099 protein groups were identified from combined GPF-DIA (cGPF-DIA, Table S5) when matching to the Arabidopsis spectral library, which represents an approximate increase of $54.7 \%$ compared to the cDIA approach ( $n=6530$, Table S1). Similarly, the numbers of peptides increased by $41.6 \%$ in cGPF-DIA (Figure 2d). When using a directDIA approach to analyze the data, we observed an apparent increase of $\sim 45.3$ and $82.3 \%$ in the number of protein groups and peptides quantified, respectively in cGPF-DIA compared to the cDIA data. This substantial increase $(82.3 \%)$ in peptide identification in directDIA analysis illustrates the importance of the high-quality MS2 spectra for DIA analysis, in particular in the absence of a comprehensive spectral library. Use of narrow precursor mass range DIA-MS enhanced MS spectra quality and thus greatly improved the peptide identification in the directDIA analysis. It is also noteworthy that matching DIA data with a spectral library generally resulted in 5-10\% more protein groups than directDIA where no preconstructed library was used. 


\section{3. cGPF-DIA Recovered Low Abundance of Proteins}

To investigate the relative abundances of identified proteins from matching Arabidopsis library, the protein intensities were log transformed with a base of 10, and plotted using histograms. Similar distributions of common identifications were observed in conventional DIA and cGPF-DIA (Figure 3a). When all identified proteins were used for the plot, the distribution of protein abundances was clearly skewed to the relatively low abundance side (Figure $3 b$ ). This suggests that the increase in protein measurement observed in the cGPFDIA method consists mainly of low abundance proteins. As a result, the dynamic range of protein identifications in CGPF-DIA was increased by $\sim 1$ order of magnitude mainly in the lower abundance range to span nearly 6 orders of magnitude. This demonstrates that a higher sensitivity can be achieved by using narrow precursor mass range DIA-MS.

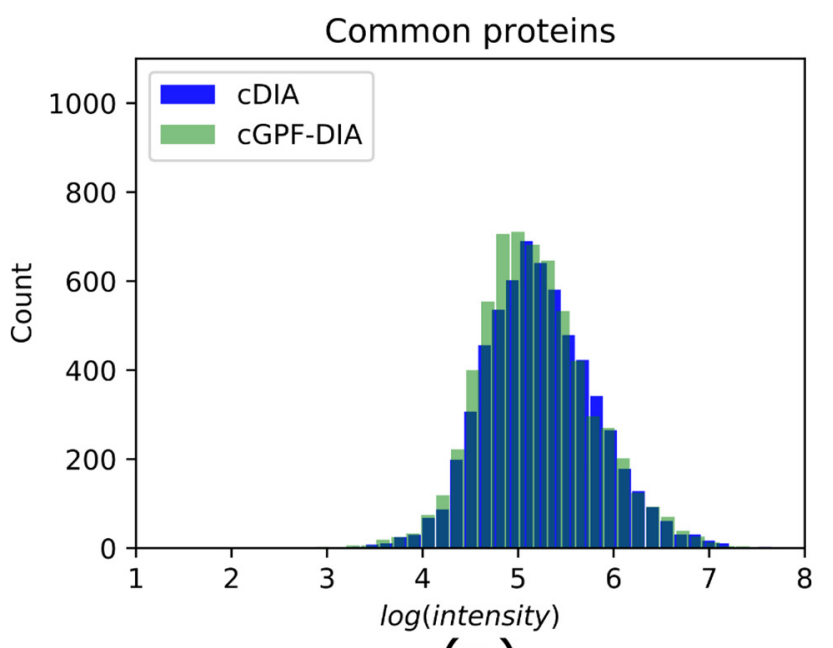

(a)

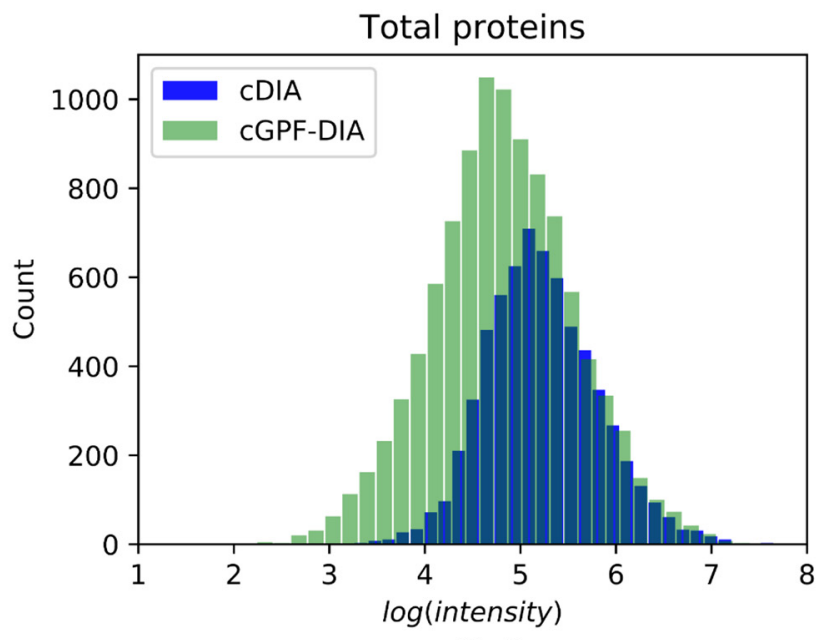

(b)

Figure 3. Histograms of the distribution of protein abundances measured in DIA experiments. (a) Distributions of common proteins identified in both cDIA (blue) and cGPF-DIA (green). Bin size is 30. The two histograms are nearly overlapping suggesting similar dynamic ranges. Most proteins have abundances from 3.2 to $7.3\left(\log _{10}(\right.$ intensity $\left.)\right)$. (b) Distributions of total protein identifications from cDIA and cGPF-DIA; the distribution of proteins in cGPF-DIA (shown in green) ranges from 2.6 to 7.3 with a clear shift to lower abundance range compared to cDIA (shown in blue) and fully encompasses the cDIA range.

\subsection{Narrow Precursor Ranges DIA Enabled Accurate Quantitation}

To assess quantitative accuracy of the workflow, every experiment was carried out three times. The median coefficient of variation $(\mathrm{CV})$ of protein group quantities was $\leq 6.5 \%$ in cDIA and $\leq 5.9 \%$ in narrow precursor range DIA, respectively. In particular, they were lower than $4.3 \%$ for the DIA with a narrow size $(250 \mathrm{~m} / \mathrm{z}$ units $)$ of precursor range, indicating higher quantitation accuracy as compared to that in cDIA.

Next, we performed a direct comparison between the cDIA and cGPF-DIA. First, we extracted common identifications $(n=6306)$ and plotted their intensities against each other (Figure 4 ). The correlation coefficient was $>0.9$ illustrating the overall similarity in quantitation achieved by the two methods. Second, the robustness of identifying differential expressions from the complex samples was demonstrated using protein mixtures containing the same amount of Arabidopsis samples $(1 \mu \mathrm{g})$ but different amounts of 6-protein mix spike-in (Table 1). As expected, there was no Arabidopsis protein identified as significantly changing in either approach, suggesting a highly accurate and reliable measurement by data independent acquisition (Figure 5). The six spike-in proteins were all identified in both approaches; however, they were successfully quantified with accurate ratios and high confidence $(Q$-value $<0.001)$ only in cGPF-DIA (Figure $5 b)$. The detailed information about their identification and quantitation are presented in Table S6 (protein information), Tables S7 and S8 (peptide information) and illustrated in supplementary material. One of the 
proteins, namely FETUB, was not found to have a significant difference due to the high $Q$-value $(Q$-value $=0.12)$ in cDIA approach (Figure 5a, Table 1$)$. In addition, the measured ratio of FETUB from the measurement of two samples by cDIA was rather inaccurate, 19.7 instead of 5 (initial mix ratio). In general, most proteins including Arabidopsis proteins were quantified at lower $Q$-value and thus with higher confidence from our narrow precursor mass range DIA.

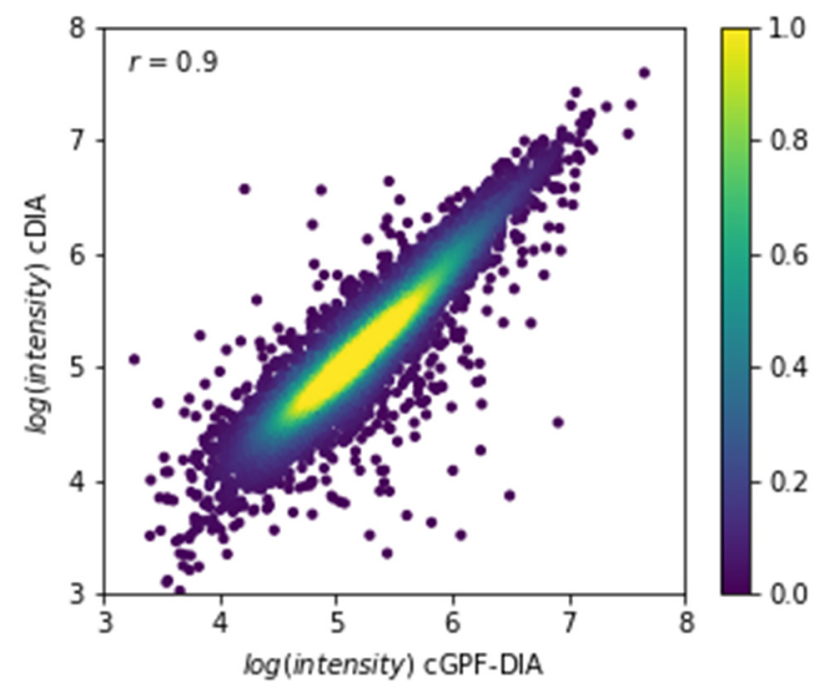

Figure 4. Correlation of quantitative intensities of common identifications $(n=6306)$ between cDIA and cGPF-DIA. The correlation coefficient was 0.9 , and the color bar illustrates the protein intensity correlation of two representative methods.

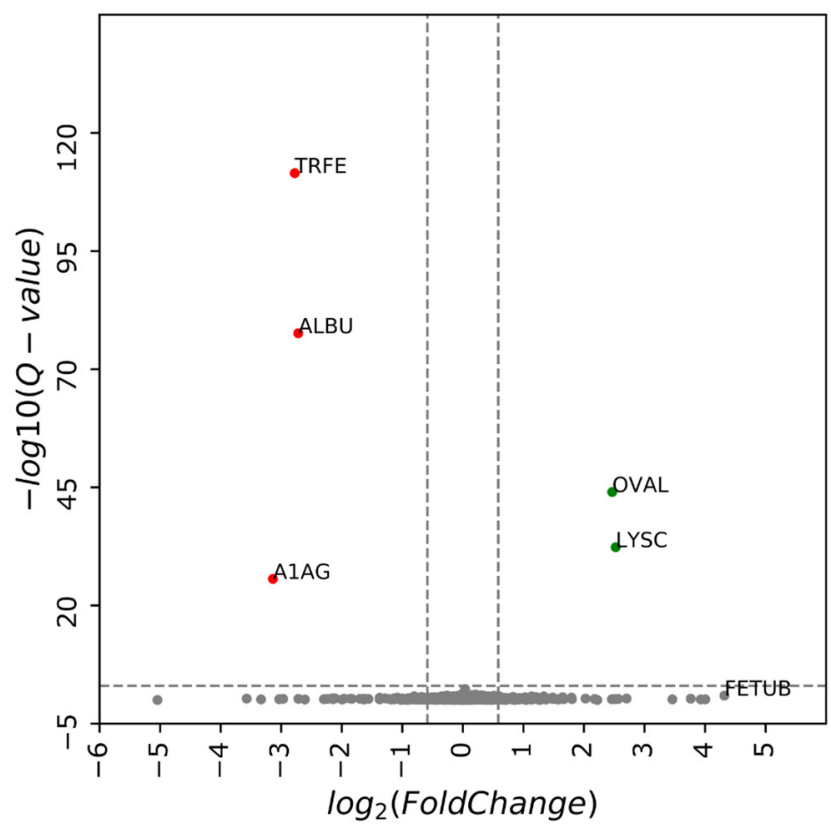

(a)

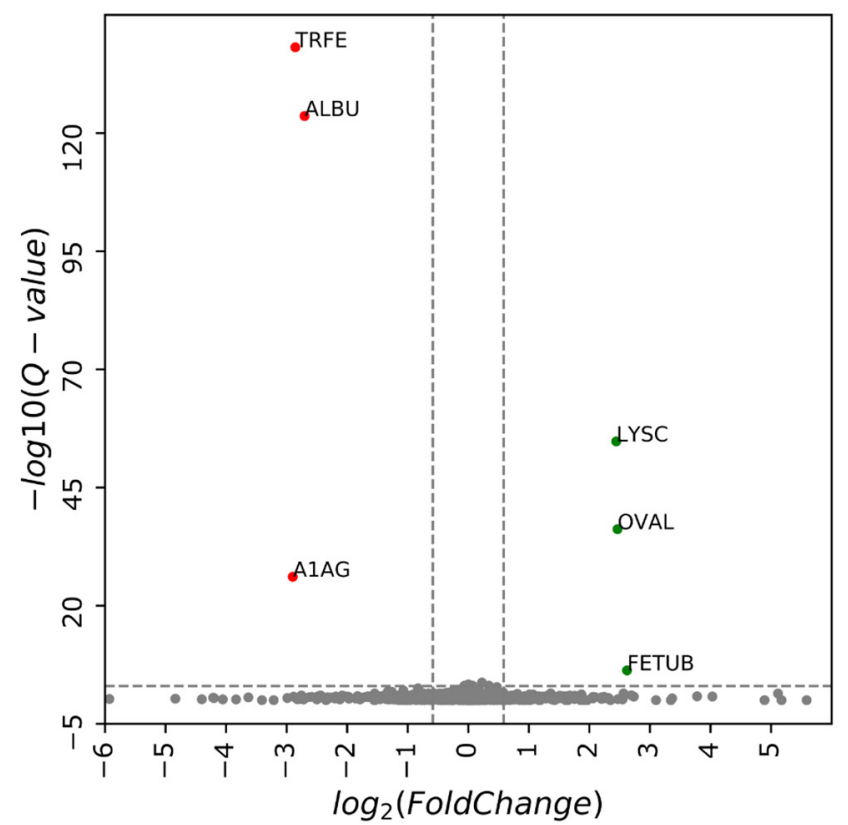

(b)

Figure 5. Volcano plot of protein intensity ratios $\log _{2}$ (samples $2 / 1$ ) obtained using (a) cDIA and (b) cGPF-DIA approaches. Proteins with a fold change (or ratio) $\geq 1.5$ and a $Q$-value $\leq 0.001$ were considered to have a significantly different expression/spike-in ratio. Shown in red are proteins that are down-regulated while green denotes up-regulated proteins. FETUB was not identified as significant differential expression in cDIA-MS due to its relatively large $Q$-value (0.12). Sample loading amounts and measured ratios of spike-in proteins are shown in Table 1. 


\section{Discussion}

Through systematic assessment of the effect of precursor mass range on the extent of protein identification and quantitative accuracy, we reported a DIA workflow of using a narrow precursor mass range that outperformed conventional DIA with a wider precursor mass range. Our method successfully identified a $34.7 \%$ higher number of protein groups with additional proteins mostly at a lower abundance range using a single injection for DIAMS of narrow precursor mass range (400-650 m/z). When combining three independent DIA-MS analyses of different narrow precursor mass range by gas-phase fractionation, a $54.7 \%$ higher number of protein groups $(n=10,099)$ could be obtained as compared to the conventional DIA approach. This represents approximately $65 \%$ of our comprehensive Arabidopsis spectral library obtained using extensive DDA runs [16]. Higher numbers of protein identification were largely attributed to the increase in spectral resolution and specificity. When using narrow precursor mass range, the isolation window for DIA is smaller and contains fewer precursor ions while the AGC target remains the same meaning that for each precursor in the range more ions can be accumulated, resulting in spectra with better signal-to-noise ratios.

Furthermore, the robustness and advantages were demonstrated for identifying differential expression in a highly complex mixture. Instead of spiking in a completely different proteome as in [19], we introduced only a few proteins in relatively low amounts into Arabidopsis root cell digest. This is to mimic real biological events where the majority of proteins remain unchanged while only a small number of proteins would respond to a stimulus. As expected, using our narrow precursor mass range DIA, all proteins were accurately quantified with high confidence while there is no falsely quantified target. In contrast, conventional DIA quantified five out of the six spiked-in proteins and failed to identify FETUB, which had a more extreme ratio $(r=19.7)$ and high $Q$-value $(Q=0.12)$.

Importantly, the workflow is simple to perform, and uses the same data analysis approach as conventional DIA. In theory, inclusion of a wide precursor m/z (400-1200) window in DIA would enable the acquisition of fragment spectra for every precursor within the defined mass range, and thus identify a near complete proteome. However, in practice, due to the limited mass spectrometry sensitivity and high dynamic range of a typical proteome, the wide precursor mass ranges usually result in chimeric spectra where low abundance ion fragments are suppressed, which prevents their identification during spectral matching. Although this issue has been long recognized and many attempts were made to address it, limited success has been achieved using overlap DIA [21,22,31]. In addition, such approaches often require the implementation of a new data analysis algorithm, thus limiting its use in the research to community.

Since the early days of DIA, a high-quality spectral library either constructed experimentally or computationally has been considered a prerequisite for good spectral matching in the DIA analysis. As such, a lot of effort has been put towards building a comprehensive library via extensive DDA analyses $[15,16,32,33]$. There are various studies showing that a sample specific library results in better identification [34,35], thus justifying the resources required for library generation. In 2017, Biognosys introduced a library-free DIA approach (coined as directDIA) which could identify up to $90 \%$ of protein groups compared to a library-based approach. Our data comparing directDIA and spectral library confirmed that an additional $5-10 \%$ increase in protein identification can be obtained in the library-based approach.

In conclusion, which DIA regimen to use will always depend on the objective of the study, the resources available and cost-benefit assessment. However, our study provides few guidelines on how to improve the number of proteins quantified in a given analysis. For example, we have shown that DIA with narrow precursor mass ranges results in an increase in quantitative accuracy while improving the protein sampling, especially at the low abundance intensity range, when compared to conventional DIA. When combining three narrow precursor ranges, covering the same range as the cDIA, we were able to maximize the number of proteins quantified, while increasing the sequence coverage of 
these proteins. This is particularly important in the study of protein isoforms, for example as a result of splicing. Furthermore, being able to access the low abundance range is very important to elucidate signaling mechanisms in various studies.

Supplementary Materials: The following are available online at https:/ / www.mdpi.com/article/ 10.3390/life11090982/s1, Table S1: Protein groups quantified using conventional data independent acquisition (cDIA); Table S2: Protein groups quantified using narrow precursor range $(400-650 \mathrm{~m} / \mathrm{z})$ data independent acquisition (DIA); Table S3: Protein groups quantified using narrow precursor range (650-900 m/z) data independent acquisition (DIA); Table S4: Protein groups quantified using narrow precursor range (900-1200 m/z) data independent acquisition (DIA); Table S5: Protein groups quantified from the combined DIA analyses of three narrow precursor ranges (400-650, 650-900 and $900-1200 \mathrm{~m} / \mathrm{z}$ ); Table S6: Protein identification and quantitation of the six spike-in proteins by cDIA and cGPF-DIA; Table S7: Peptide identification and quantitation of the six spike-in proteins by cDIA-MS; Table S8: Peptide identification and quantitation of spike-in proteins by cGPF-DIA-MS. Supplementary text and figures.

Author Contributions: Conceptualization, H.Z. and D.B.; methodology and formal analysis, H.Z.; writing—original draft preparation, H.Z. and D.B.; writing—review and editing, H.Z. and D.B. All authors have read and agreed to the published version of the manuscript.

Funding: This research used resources provided by the KAUST Core Laboratories.

Institutional Review Board Statement: Not applicable.

Informed Consent Statement: Not applicable.

Data Availability Statement: The mass spectrometry proteomics data together with the analyses using Spectronaut software have been deposited to the ProteomeXchange Consortium via the PRIDE [1] partner repository with the dataset identifier PXD026965.

Acknowledgments: We thank the director of the Bioscience core laboratory, Nicole Cheung, for her support in this project.

Conflicts of Interest: The authors declare no conflict of interest.

\section{References}

1. Washburn, M.P.; Wolters, D.; Yates, J.R. Large-scale analysis of the yeast proteome by multidimensional protein identification technology. Nat. Biotechnol. 2001, 19, 242-247. [CrossRef] [PubMed]

2. Zhao, C.; Zhang, H.; Wong, W.C.; Sem, X.; Han, H.; Ong, S.M.; Tan, Y.C.; Yeap, W.H.; Gan, C.S.; Ng, K.Q.; et al. Identification of novel functional differences in monocyte subsets using proteomic and transcriptomic methods. J. Proteome Res. 2009, 8, $4028-4038$. [CrossRef] [PubMed]

3. Chan, Y.K.; Zhang, H.; Liu, P.; Tsao, S.W.; Lung, M.L.; Mak, N.K.; Ngok-Shun Wong, R.; Ying-Kit Yue, P. Proteomic analysis of exosomes from nasopharyngeal carcinoma cell identifies intercellular transfer of angiogenic proteins. Int. J. Cancer 2015, 137, 1830-1841. [CrossRef] [PubMed]

4. He, T.; Jin Kim, Y.; Heidbrink, J.L.; Moore, P.A.; Ruben, S.M. Drug target identification and quantitative proteomics. Expert Opin. Drug Discov. 2006, 1, 477-489. [CrossRef]

5. Wang, J.; Wong, Y.K.; Zhang, J.; Lee, Y.M.; Hua, Z.C.; Shen, H.M.; Lin, Q. Drug Target Identification Using an iTRAQ-Based Quantitative Chemical Proteomics Approach-Based on a Target Profiling Study of Andrographolide. Methods Enzymol. 2017, 586, 291-309. [CrossRef] [PubMed]

6. Amiri-Dashatan, N.; Ahmadi, N.; Rezaei-Tavirani, M.; Koushki, M. Identification of differential protein expression and putative drug target in metacyclic stage of Leishmania major and Leishmania tropica: A quantitative proteomics and computational view. Comp. Immunol. Microbiol. Infect. Dis. 2021, 75, 101617. [CrossRef]

7. Unwin, R.D.; Pierce, A.; Watson, R.B.; Sternberg, D.W.; Whetton, A.D. Quantitative proteomic analysis using isobaric protein tags enables rapid comparison of changes in transcript and protein levels in transformed cells. Mol. Cell. Proteom. 2005, 4, 924-935. [CrossRef]

8. Thompson, A.; Schafer, J.; Kuhn, K.; Kienle, S.; Schwarz, J.; Schmidt, G.; Neumann, T.; Johnstone, R.; Mohammed, A.K.; Hamon, C. Tandem mass tags: A novel quantification strategy for comparative analysis of complex protein mixtures by MS/MS. Anal. Chem. 2003, 75, 1895-1904. [CrossRef]

9. Ong, S.E.; Blagoev, B.; Kratchmarova, I.; Kristensen, D.B.; Steen, H.; Pandey, A.; Mann, M. Stable isotope labeling by amino acids in cell culture, SILAC, as a simple and accurate approach to expression proteomics. Mol. Cell. Proteom. 2002, 1, 376-386. [CrossRef] 
10. Chelius, D.; Bondarenko, P.V. Quantitative profiling of proteins in complex mixtures using liquid chromatography and mass spectrometry. J. Proteome Res. 2002, 1, 317-323. [CrossRef]

11. Ow, S.Y.; Salim, M.; Noirel, J.; Evans, C.; Rehman, I.; Wright, P.C. iTRAQ underestimation in simple and complex mixtures: The good, the bad and the ugly. J. Proteome Res. 2009, 8, 5347-5355. [CrossRef]

12. Gallien, S.; Duriez, E.; Crone, C.; Kellmann, M.; Moehring, T.; Domon, B. Targeted proteomic quantification on quadrupoleorbitrap mass spectrometer. Mol. Cell. Proteom. 2012, 11, 1709-1723. [CrossRef]

13. Kuhn, E.; Wu, J.; Karl, J.; Liao, H.; Zolg, W.; Guild, B. Quantification of C-reactive protein in the serum of patients with rheumatoid arthritis using multiple reaction monitoring mass spectrometry and 13C-labeled peptide standards. Proteomics 2004, 4, 1175-1186. [CrossRef]

14. Gillet, L.C.; Navarro, P.; Tate, S.; Rost, H.; Selevsek, N.; Reiter, L.; Bonner, R.; Aebersold, R. Targeted data extraction of the MS/MS spectra generated by data-independent acquisition: A new concept for consistent and accurate proteome analysis. Mol. Cell. Proteom. 2012, 11, 1-17. [CrossRef] [PubMed]

15. Rosenberger, G.; Koh, C.C.; Guo, T.; Rost, H.L.; Kouvonen, P.; Collins, B.C.; Heusel, M.; Liu, Y.; Caron, E.; Vichalkovski, A.; et al. A repository of assays to quantify 10,000 human proteins by SWATH-MS. Sci. Data 2014, 1, 140031. [CrossRef] [PubMed]

16. Zhang, H.; Liu, P.; Guo, T.; Zhao, H.; Bensaddek, D.; Aebersold, R.; Xiong, L. Arabidopsis proteome and the mass spectral assay library. Sci. Data 2019, 6, 278. [CrossRef] [PubMed]

17. Gessulat, S.; Schmidt, T.; Zolg, D.P.; Samaras, P.; Schnatbaum, K.; Zerweck, J.; Knaute, T.; Rechenberger, J.; Delanghe, B.; Huhmer, A.; et al. Prosit: Proteome-wide prediction of peptide tandem mass spectra by deep learning. Nat. Methods 2019, 16, 509-518. [CrossRef]

18. Bekker-Jensen, D.B.; Bernhardt, O.M.; Hogrebe, A.; Martinez-Val, A.; Verbeke, L.; Gandhi, T.; Kelstrup, C.D.; Reiter, L.; Olsen, J.V. Rapid and site-specific deep phosphoproteome profiling by data-independent acquisition without the need for spectral libraries. Nat. Commun. 2020, 11, 787. [CrossRef]

19. Bruderer, R.; Bernhardt, O.M.; Gandhi, T.; Xuan, Y.; Sondermann, J.; Schmidt, M.; Gomez-Varela, D.; Reiter, L. Optimization of Experimental Parameters in Data-Independent Mass Spectrometry Significantly Increases Depth and Reproducibility of Results. Mol. Cell. Proteom. 2017, 16, 2296-2309. [CrossRef] [PubMed]

20. Zhang, Y.; Bilbao, A.; Bruderer, T.; Luban, J.; Strambio-De-Castillia, C.; Lisacek, F.; Hopfgartner, G.; Varesio, E. The Use of Variable Q1 Isolation Windows Improves Selectivity in LC-SWATH-MS Acquisition. J. Proteome Res. 2015, 14, 4359-4371. [CrossRef]

21. Amodei, D.; Egertson, J.; MacLean, B.X.; Johnson, R.; Merrihew, G.E.; Keller, A.; Marsh, D.; Vitek, O.; Mallick, P.; MacCoss, M.J. Improving Precursor Selectivity in Data-Independent Acquisition Using Overlapping Windows. J. Am. Soc. Mass Spectrom. 2019, 30, 669-684. [CrossRef]

22. Borras, E.; Sabido, E. DIA+: A Data-Independent Acquisition Method Combining Multiple Precursor Charges to Improve Peptide Signal. Anal. Chem. 2018, 90, 12339-12341. [CrossRef]

23. Cai, X.; Ge, W.; Yi, X.; Sun, R.; Zhu, J.; Lu, C.; Sun, P.; Zhu, T.; Ruan, G.; Yuan, C.; et al. PulseDIA: Data-Independent Acquisition Mass Spectrometry Using Multi-Injection Pulsed Gas-Phase Fractionation. J. Proteome Res. 2021, 20, 279-288. [CrossRef] [PubMed]

24. Ge, W.; Liang, X.; Zhang, F.; Xu, L.; Xiang, N.; Sun, R.; Liu, W.; Xue, Z.; Yi, X.; Wang, B.; et al. Optimization of Spectral Library Size Improves DIA-MS Proteome Coverage. bioRxiv 2020. [CrossRef]

25. Searle, B.C.; Swearingen, K.E.; Barnes, C.A.; Schmidt, T.; Gessulat, S.; Kuster, B.; Wilhelm, M. Generating high quality libraries for DIA MS with empirically corrected peptide predictions. Nat. Commun. 2020, 11, 1548. [CrossRef]

26. Meier, F.; Brunner, A.D.; Frank, M.; Ha, A.; Bludau, I.; Voytik, E.; Kaspar-Schoenefeld, S.; Lubeck, M.; Raether, O.; Bache, N.; et al. diaPASEF: Parallel accumulation-serial fragmentation combined with data-independent acquisition. Nat. Methods 2020, 17, 1229-1236. [CrossRef] [PubMed]

27. Hebert, A.S.; Prasad, S.; Belford, M.W.; Bailey, D.J.; McAlister, G.C.; Abbatiello, S.E.; Huguet, R.; Wouters, E.R.; Dunyach, J.J.; Brademan, D.R.; et al. Comprehensive Single-Shot Proteomics with FAIMS on a Hybrid Orbitrap Mass Spectrometer. Anal. Chem. 2018, 90, 9529-9537. [CrossRef]

28. Ordonez, N.M.; Marondedze, C.; Thomas, L.; Pasqualini, S.; Shabala, L.; Shabala, S.; Gehring, C. Cyclic mononucleotides modulate potassium and calcium flux responses to H2O2 in Arabidopsis roots. FEBS Lett. 2014, 588, 1008-1015. [CrossRef]

29. Wisniewski, J.R.; Zougman, A.; Nagaraj, N.; Mann, M. Universal sample preparation method for proteome analysis. Nat. Methods 2009, 6, 359-362. [CrossRef] [PubMed]

30. Zhang, H.; Qian, P.Y.; Ravasi, T. Selective phosphorylation during early macrophage differentiation. Proteomics 2015, 15, 3731-3743. [CrossRef]

31. Guan, S.; Taylor, P.P.; Han, Z.; Moran, M.F.; Ma, B. Data Dependent-Independent Acquisition (DDIA) Proteomics. J. Proteome Res. 2020, 19, 3230-3237. [CrossRef]

32. Yang, Y.; Liu, X.; Shen, C.; Lin, Y.; Yang, P.; Qiao, L. In silico spectral libraries by deep learning facilitate data-independent acquisition proteomics. Nat. Commun. 2020, 11, 146. [CrossRef] [PubMed]

33. Sim, K.H.; Liu, L.C.; Tan, H.T.; Tan, K.; Ng, D.; Zhang, W.; Yang, Y.; Tate, S.; Bi, X. A comprehensive CHO SWATH-MS spectral library for robust quantitative profiling of 10,000 proteins. Sci. Data 2020, 7, 263. [CrossRef] [PubMed] 
34. Lou, R.; Tang, P.; Ding, K.; Li, S.; Tian, C.; Li, Y.; Zhao, S.; Zhang, Y.; Shui, W. Hybrid Spectral Library Combining DIA-MS Data and a Targeted Virtual Library Substantially Deepens the Proteome Coverage. iScience 2020, 23, 100903. [CrossRef] [PubMed]

35. Pak, H.; Michaux, J.; Huber, F.; Chong, C.; Stevenson, B.J.; Muller, M.; Coukos, G.; Bassani-Sternberg, M. Sensitive Immunopeptidomics by Leveraging Available Large-Scale Multi-HLA Spectral Libraries, Data-Independent Acquisition, and MS/MS Prediction. Mol. Cell. Proteom. 2021, 20, 100080. [CrossRef] 